\title{
Topical microbicides against HIV spread: what, where and why?
}

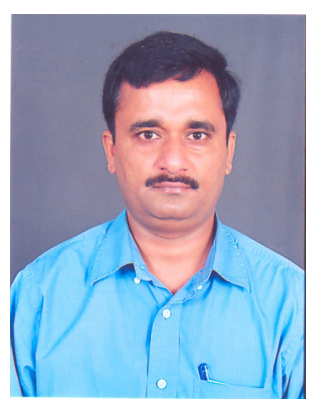

Sunit Kumar Singh Center for Cellular \& Molecular Biology (CCMB), Room S107, Section of Infectious Diseases, Uppal Road, Hyderabad, 500007, India

Tel.: +914027192523; Fax: +914027160591; sunitsingh@ccmb.res.in; sunitsingh2000@gmail.com

$$
\begin{aligned}
& \text { 'Topical microbicides are } \\
& \text { self-administered prophylactic } \\
& \text { agents ... one of the most } \\
& \text { promising technologies under } \\
& \text { development to reduce the risk } \\
& \text { of contracting sexually } \\
& \text { transmitted infections.' }
\end{aligned}
$$

Worldwide there are an estimated 40 million people living with HIV/AIDS. The HIV pandemic is growing around the globe and women of reproductive age face a high risk of HIV infection. In Sub-Saharan Africa approximately $8 \%$ of adults are infected with HIV, with approximately $60 \%$ of all infections observed among women [1]. Women's risk of contracting HIV continues to be high despite the relatively low chances of male to female HIV transmission [2-4]. Heterosexual transmission is influenced by several key epidemiological factors such as age, gender, mobility and the presence of other sexually transmitted infections. Vulnerability in women increases because of a lack of economic and social power in many societies, where women often cannot control sexual encounters or feel unable to insist on protective measures such as condoms or mutual monogamy. There is a clear need for new technologies that women can use and control, to reduce their risk of acquiring sexually transmitted infections. According to the present scenario, the development of a vaccine against HIV will take many years. In the meantime, the scientific community could apply and develop other biological methods to halt the transmission of HIV. Microbicides have long had a 'stepchild' treatment in the AIDS research community. Industry has had little interest in developing a topical gel or cream that can halt the transmission of HIV through the vagina or rectum. Over the past few years, however, nonprofit organizations and governments have provided substantial money to microbicide research and development.

\section{What are topical microbicides?}

Topical microbicides are self-administered prophylactic agents that could be applied to the vagina or rectum in various formulations. They are one of the most promising technologies under development to reduce the risk of contracting sexually transmitted diseases. Microbicides can be protective not only against viruses, such as HIV and genital herpes, but also against common bacterial infections, such as gonorrhea, chlamydia and other sexually transmitted pathogens. I will discuss here the role of microbicides in the prevention of HIV transmission. The basic idea is simple but the development and evaluation is highly complex. Anti-infective chemicals, selected from a great variety of substances that are known to block HIV and other sexually transmitted pathogens, are formulated to create products suitable for insertion into the vagina before intercourse [5]. These microbicides can be in various forms, such as gels, creams, foams, impregnated sponges, suppositories or films [5].

'...prevention efforts have centered on three approaches: behavioral change (safer sex), development of a vaccine and development of a microbicide.'

When discussing the most prevalent sexually transmitted disease, HIV, we find that prevention efforts have centered on three approaches: behavioral change (safer sex), development of a vaccine and development of a microbicide.

\section{What should microbicides do?}

The main aim of microbicides is to reduce male to female HIV transmission. Microbicides could potentially prevent both male to female and female to male transmission. Some microbicides can also act as a contraceptive alongside their role in the prevention of the transmission of sexually transmitted pathogens [6,7]. Microbicides must be able to do one or more of the following in order to prevent HIV infection (Figure 1): assist the natural defenses in the vagina to inactivate the virus; inactivate the virus while it is in the vagina; prevent the virus from attaching to and fusing with host cells in the genital tract and prevent the virus from replicating if it enters the host cells successfully. 


\section{HIV life cycle (in general)}

Improved understanding of the role of the HIV life cycle (binding, fusion, reverse transcription, DNA integration, host-cell activation, transcription, translation, protein assembly, budding and viral release), in the mechanism of HIV infection and transmission across the mucosal epithelium and the tropism of different viral strains provides new possibilities for antiviral interventions. This includes the recognition of the role of coreceptors in HIV binding and the particular role that dendritic cells (DCs) play in transmitting the virus to the lymphatic system. In addition to the high affinity of the viral envelope glycoprotein, gp120, for binding to the CD4 protein on the host cell, a coreceptor, for example, the CCR5 used by macrophage tropic strains or the CXCR4 used by T-cell tropic strains, is required for HIV to enter the cell. The binding of gp120 with receptors and coreceptors triggers a conformational change in the viral envelope that leads to fusion of the virus and host cell membranes and entry of the viral genome into the host cell. DCs, such as the Langerhans cells of the skin and mucosal membrane and the follicular DCs of lymphoid tissue, capture antigens and transport them to $\mathrm{T}$ cells. The majority of DCs also carry CXCR4, DC-specific intracellular cell adhesion molecule-grabbing nonintegrin and CCR5 that effectively bind HIV through gp120.
Infected DCs can migrate to local lymph nodes, where extensive HIV replication takes place, leading to generalized systemic infection.

The potential sources of transmissible HIV are free virus particles, infected lymphoid cells in semen, cervicovaginal secretions, blood or other fluids present as a result of physical trauma or genital infections. The epithelium of the vagina and external surface of the cervix is multilayered and is relatively strong and durable. Beneath, there is a layer of connective tissue known as the lamina propria.

\section{'Anti-infective chemicals ... are formulated to create products suitable for insertion into the vagina before intercourse.'}

Within these structures are several classes of immune cells, such as lymphoid cells, including DCs, macrophages, $T$ lymphocytes and Langerhans cells. The nonlymphoid squamous cells, which comprise the multilayered genital epithelium, have none of the necessary receptors for HIV and appear to be resistant to infection [5]. These cells do not allow the virus to migrate through them by passive transcytosis, as in the case of the epithelium lining the gastrointestinal tract. So how does the virus colonize here?

\section{Figure 1. Diagram of the mode of action of topical vaginal microbicides.}

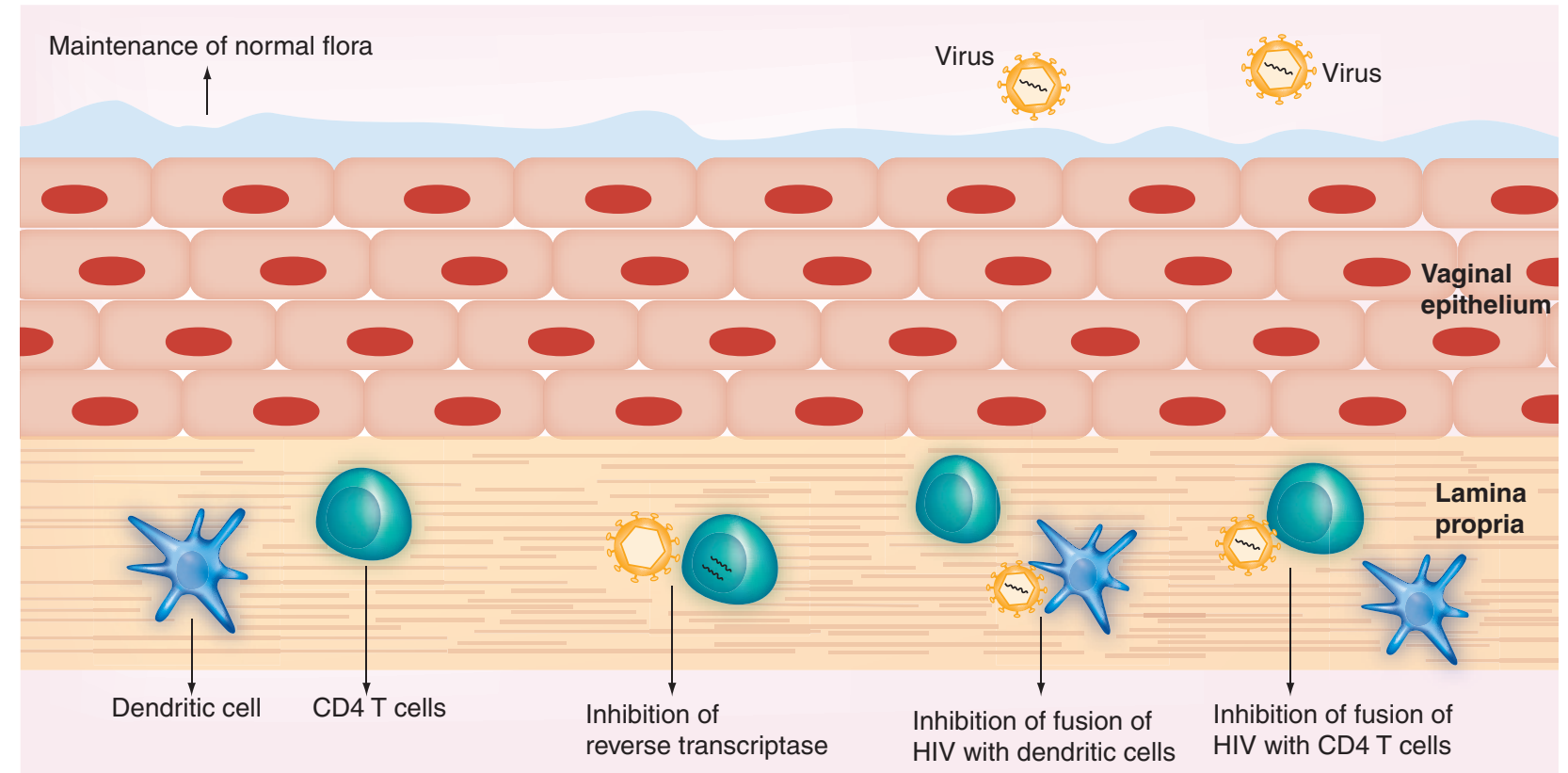

Topical vaginal microbicides could maintain the normal vaginal flora. They can target HIV fusion and adsorption with dendritic and CD4 T cells. Microbicides can also target virus reverse transcriptase activity and prevent integration into the host cell genome. 
There could be two ways: either, Langerhan cells and $\mathrm{T}$ lymphocytes in the epithelium bind the virus and migrate to the underlying layer of the lamina propria, or, HIV can penetrate its subepithelial cells through physical breaches in epithelial integrity [5].

We are currently in urgent need of some form of chemical barrier to prevent the sexual transmission of HIV through the vaginal route. Topical microbicides appear to be one of the most effective options. We can divide the topical microbicides (Table 1) into two broad categories for easy understanding: nonspecific microbicides and specific microbicides.

\section{Nonspecific microbicides}

The nonspecific microbicides consist of buffering agents, detergents and surfactants that include nonionic, anionic or cationic compounds. Detergents destroy the viral envelope by solubilizing the membrane proteins. However, this nonspecific mechanism of action may disrupt the cell membranes of the vaginal and cervical epithelium and cause erosions and lesions, leading to an increased risk of infection. Surfactants, for example, nonoxynol-9, also work as spermicides by disaggregating the lipid membranes of spermatozoa. As discussed above, clinical trial studies reported that nonoxynol-9 is responsible for genital ulcers in some women. In 2002, WHO experts concluded that this substance should not be used as a microbicide. The buffering agents (Buffer-Gel ${ }^{\circledR}$ and Acidform ${ }^{\mathrm{TM}}$ ) also fall under the category of nonspecific products. They act by

Table 1. Microbicides and their mode of action.

\begin{tabular}{|c|c|}
\hline Name of micobicide & Mode of action \\
\hline $\operatorname{Savvy}^{\mathrm{TM}}(\mathrm{C} 31 \mathrm{G})$ & Membrane disruption \\
\hline MucoCept & Defense enhancer \\
\hline Lime juice & Defense enhancer \\
\hline Acidform ${ }^{\mathrm{TM}}$ & Defense enhancer \\
\hline Buffer-Gel ${ }^{\circledR}$ & Defense enhancer \\
\hline Tenofovir & Reverse transcriptase inhibitor \\
\hline TMC-120 & Reverse transcriptase inhibitor \\
\hline UC-781 & Reverse transcriptase inhibitor \\
\hline Carraguard ${ }^{\circledR}$ & Reverse transcriptase inhibitor \\
\hline Cellulose sulfate & Entry fusion inhibitor \\
\hline Cyanovirin & Entry fusion inhibitor \\
\hline PRO2000 & Entry fusion inhibitor \\
\hline Novaflux & Entry fusion inhibitor \\
\hline PSC-RANTES & Entry fusion inhibitor \\
\hline MAb b12 & Entry fusion inhibitor \\
\hline
\end{tabular}

affecting a local $\mathrm{pH}$ change that results in the inactivation of the microorganisms. These buffering agents are designed to supplement or enhance the natural immune defences of the vagina. Buffer-Gel ${ }^{\circledR}$ reduces and retains the vaginal $\mathrm{pH}$ at a low level even after the arrival of alkaline ejaculate. It has completed the Phase I trial successfully [8]. Combinations of microbiological, chemical and physical barriers are required to protect the vagina from sexually transmitted infections. Lactobacilli occur as a natural flora in the vagina and release a variety of antimicrobial compounds, such as lactic acid, hydrogen peroxide and bacteriocins. The vagina is usually maintained at a low $\mathrm{pH}$ of approximately 4 . This low $\mathrm{pH}$ is achieved through the secretion of lactic acid by lactobacilli that colonize the vagina. These lactobacilli are sometimes destroyed by other vaginal infections, leading to bacterial vaginosis. The destruction of lactobacilli leads to changes in $\mathrm{pH}$ and makes the vagina prone to other sexually transmitted infections.

\section{'...buffering agents ... act by affecting a local pH change that results in the inactivation of the microorgansims.'}

Microbicides have been developed to maintain the colonization of the vagina by lactobacilli or to recolonize the vagina with lactobacilli when these commensal organisms have been adversely affected, for example, by the use of antibiotics or by genital-tract infections.

\section{Specific microbicides}

Microbicides with specific functions belong to the category of specific microbicides. Some potential agents, such as C31G $\left(\right.$ Savvy $\left.^{\mathrm{TM}}\right)$, are under clinical-trial studies. Another naphthalene sulphonate polymer, PRO 2000, has a far better therapeutic index. These substances also retain their antiviral activities in the presence of semen [9]. These anionic polymers can inhibit infection by free virus particles and also block transmission by HIV-infected lymphoid cells [10]. A variety of anionic substances that target the adsorption and fusion process of the virus belong to this category. Anionic polymers do not physically destroy HIV as do the surfactants but instead work by preventing the attachment-fusion step of the infection process. Most of them bind to the viral envelope through the negative charges and block cell entry. These can be effective not only in HIV but also in other enveloped viruses, such as herpes viruses. Anionic products are the most 
advanced of the microbicides in clinical development. One product, Carraguard ${ }^{\circledR}$, is in Phase III of an HIV-prevention trial. HIV-specific microbicides should preferentially block the viral replication cycle at a step before integration of the proviral genome into the target cell. Several phases of the virus life cycle can be targeted for microbicidal intervention.

$\therefore$...anionic substances that target the adsorption and fusion process of the virus ... are the most advanced of the microbicides in clinical development.'

The relatively high molecular masses of these substances means that they are poorly absorbed through the vaginal epithelium, reducing the risk of systemic toxicity. Several anionic polymers have been demonstrated to be protective, to varying degrees, against vaginal challenge with simian immunodeficiency virus or simian HIV in the macaque. These include dextrin-2-sulphate [9], PRO 2000 [5,9,11] and cellulose acetate phthalate [12]. PRO 2000 [11] and carrageenan [13] have also been shown to protect against the genital herpes virus in mouse models.

Along with the above mentioned products of different origins, small interfering RNAs (siRNAs) have also been reported to act as microbicides. Several papers have shown that mucosal tissues are efficient at taking up siRNA. Now, scientists have started to explore the possibility of delivering siRNAs into vaginal tissues to protect against sexually transmitted pathogens. In a recent paper published in Nature, Judy Lieberman and colleagues describe an siRNA-based microbicide that proved effective against vaginal herpes simplex virus (HSV)-2 challenge in a mouse model [14]. As evaluated by clinical disease scoring and by survival, the treatment regimen resulted in highly significant protection. There was no evidence of inflammation caused by siRNA alone or for the emergence of viral escape mutants after treatment. Even post-exposure treatment ( 3 and $6 \mathrm{~h}$ after viral challenge) with a combination of two different HSV-2-specific siRNAs conferred significant protection. This is an important finding given that one of the main problems with current microbicides is their requirement to be administered before sexual intercourse.

When discussing the primary risk factors for HIV transmission, we find that, along with unprotected vaginal intercourse, unprotected anal intercourse is also an important contributory factor for HIV spread. A recent study of almost
13,000 heterosexual Americans documented a $35-40 \%$ prevalence of ever having practiced anal intercourse $[15,16]$. The rectal mucosa is extremely vulnerable to HIV infection and products with a reasonable safety profile in the vagina may cause major problems in the rectum. The rectal and vaginal compartments differ with respect to anatomy, histology, microbiology and physiology. On the one hand, the vagina is a relatively confined space, whereas the colon is an open-ended tube extending from the anus to the small intestine. As a consequence, there is a clear need to integrate rectal safety studies into microbicide development studies in order to prove their utility as rectal microbicides. The evaluation of candidate microbicides for rectal safety is in progress but rectal-safety studies are highly limited.

\section{Concerns \& other issues}

Successful microbicides would have to fulfill some important criteria regarding safety, acceptability, efficacy and affordability. Safety is a big concern. The field of microbicides suffered a significant setback when efficacy trials of surfactant nonoxynol-9 demonstrated that the HIV-1 transmission rate was greater in the active group than in the placebo group [17].

\section{If microbicides are used ... it will be important to determine whether they affect localized immune responses to vaccines.'}

It was later found that repetitive use of nonoxynol-9 disrupted the vaginal epithelium, damaging an important natural barrier against HIV [17]. It is therefore important to test carcinogenicity and teratogenicity over the long term. If microbicides are used in the same populations targeted by vaccine trials, it will be important to determine whether they affect localized immune responses to vaccines. Microbicides should not cause localized inflammation because migration of immune cells to inflammation sites would probably facilitate viral transmission [18-20]. Efficacy is the second factor for consideration. Adverse effects on epithelial integrity and immune activation can also impair efficacy. There are also issues of public perception. Multiple sequential failures may compromise the prospects of future trials of a more promising concept. A recent report on the microbicide, Ushercell (vaginal gel), has shown that it increases the rate of HIV transmission instead of preventing the transmission. In earlier trials, Ushercell was proved to act as an entry inhibitor. 
Cellulose sulphate is the active ingredient found in this vaginal gel, which binds to the viral envelope proteins and prevents them from fusing with the host cells. Ushercell appeared to be both safe and effective. However, in a large Phase III trial of 1333 sexually active women in South Africa, Uganda and India, the researchers discovered that more women using this gel had become infected with HIV than those using the placebo. This report swiftly put an early end to the trial [21]. As mentioned, Carraguard, a seaweed extract that also works as an entry inhibitor, is undergoing Phase III trial. The clinical trial study enrolled 7000 participants and found a 33\% decrease in HIV incidence. So, neither Carraguard nor Ushercell have been reported to be $100 \%$ effective in Phase III trials. Ushercell's failure has bolstered skepticism about the use of microbicides [21]. It is also important to consider the affordability of microbicides under development. Affordability is relevant because a microbicide must be manufactured at a cost that allows the product to be accessible to users in the developing world.

\section{Conclusion}

Microbicide research is undergoing a period of rapid development. It is expected that clinical trials of first generation products should be over within
1-2 years. Meanwhile, research is ongoing to identify new candidates as microbicides and to improve formulations and develop combination products [22]. A critical step will be to develop products that do not have to be used in a coitallydependent fashion. Currently, microbicides and vaccines are viewed as two very different modalities in the area of HIV prevention. However, microbicides may be used to deliver immunogens topically and function effectively as mucosal vaccines.

There is a clear requirement for topical products to prevent HIV spread. Acceptability studies among men and women in several countries show that microbicides would be acceptable. The development of microbicides as a protection mechanism for women remains hopeful and provides real potential to influence the course of the HIV epidemic. Even after a safe and effective vaccine is discovered, vaccines and microbicides will have different, but complementary approaches towards a global HIV-prevention strategy. We have to solve not only the scientific challenges but also many societal issues in terms of microbicide development. The level of funding should be increased in microbicide research. The advocacy for microbicides should be intensified so that health-policy officials and end-users are properly informed.

\section{Bibliography}

1. Myer L, Kuhn L, Stein ZA, Wright TC Jr, Denny L: Intravaginal practices, bacterial vaginosis, and women's susceptibility to HIV infection: epidemiological evidence and biological mechanisms. Lancet Infect. Dis. 5(12), 786-794 (2005).

2. Padian NS, Shiboski SC, Jewell NP: The effect of number of exposures on the risk of heterosexual HIV transmission. J. Infect. Dis. 161(5), 883-887 (1990).

3. Royce RA, Sena A, Cates W Jr, Cohen MS: Sexual transmission of HIV. N. Engl. J. Med. 336(15), 1072-1078 (1997).

4. de Vincenzi I: A longitudinal study of human immunodeficiency virus transmission by heterosexual partners. European Study Group on Heterosexual Transmission of HIV. N. Engl. J. Med. 331(6), 341-346 (1994).

5. Stone A: Microbicides: a new approach to preventing HIV and other sexually transmitted infections. Nat. Rev. Drug Discov. 1(12), 977-985 (2002).

6. Elias CJ, Coggins C: Female-controlled methods to prevent sexual transmission of HIV. AIDS 10(Suppl. 3), S43-S51 (1996).
7. Elias CJ, Heise LL: Challenges for the development of female-controlled vaginal microbicides. AIDS 8(1), 1-9 (1994).

8. Mayer KH, Peipert J, Fleming T et al: Safety and tolerability of BufferGel ${ }^{\circledR}$, a novel vaginal microbicide, in women in the United States. Clin. Infect Dis. 32(3), 476-482 (2001).

9. Weber J, Nunn A, O'Connor T et al: 'Chemical condoms' for the prevention of HIV infection: evaluation of novel agents against SHIV(89.6PD) in vitro and in vivo. AIDS 15(12), 1563-1568 (2001).

10. Javan CM, Gooderham NJ, Edwards RJ, Davies DS, Shaunak S: Anti-HIV type 1 activity of sulfated derivatives of dextrin against primary viral isolates of HIV type 1 in lymphocytes and monocyte-derived macrophages. AIDS Res. Hum. Retroviruses 13(10), 875-880 (1997).

11. Bourne N, Bernstein DI, Ireland J, Sonderfan AJ, Profy AT, Stanberry LR: The topical microbicide PRO 2000 protects against genital herpes infection in a mouse model. J. Infect. Dis. 180(1), 203-205 (1999).

12. Manson KH, Wyand MS, Miller C, Neurath AR: Effect of a cellulose acetate phthalate topical cream on vaginal transmission of simian immunodeficiency virus in rhesus monkeys. Antimicrob. Agents Chemother. 44(11), 3199-3202 (2000).

13. Zacharopoulos VR, Phillips DM: Vaginal formulations of carrageenan protect mice from herpes simplex virus infection. Clin. Diagn. Lab. Immunol. 4(4), 465-468 (1997).

14. Palliser D, Chowdhury D, Wang QY et al.: An siRNA-based microbicide protects mice from lethal herpes simplex virus 2 infection. Nature 439(7072), 89-94 (2006).

15. Mosher WD, Chandra A, Jones J: Sexual behavior and selected health measures: men and women 15-44 years of age, United States 2002. Adv. Data (362), $1-55$ (2005).

16. McGowan I: Microbicides: a new frontier in HIV prevention. Biologicals 34(4), 241-255 (2006).

17. Jain JK, Li A, Minoo P, Nucatola DL, Felix JC: The effect of nonoxynol-9 on human endometrium. Contraception 71(2), 137-142 (2005).

18. Klasse PJ, Shattock RJ, Moore JP: Which topical microbicides for blocking HIV-1 transmission will work in the real world? PLoS Med. 3(9) (2006) (Epub ahead of print). 
19. Gupta K, Klasse PJ: How do viral and host factors modulate the sexual transmission of HIV? Can transmission be blocked? PLoS Med. 3(2), e79 (2006).

20. Haase AT: Perils at mucosal front lines for HIV and SIV and their hosts. Nat. Rev Immunol. 5(10), 783-792 (2005).
21. Bolognesi N: AIDS gel's failure calls prevention approach into question. Nat. Med. 13(3), 230 (2007).

22. Veazey RS, Springer MS, Marx PA, Dufour J, Klasse PJ, Moore JP: Protection of macaques from vaginal SHIV challenge by an orally delivered CCR5 inhibitor. Nat. Med. 11(12), 1293-1294 (2005).

\section{Affiliation}

- Sunit Kumar Singh, PhD Center for Cellular \& Molecular Biology (CCMB), Room S107, Section of Infectious Diseases, Uppal Road, Hyderabad 500007, India Tel.: + 914027192523 ; Fax: +914027160591 ; sunitsingh@ccmb.res.in; sunitsingh2000@gmail.com 